\title{
Effects of ac-field amplitude on the dielectric susceptibility of relaxors
}

\author{
Zhi-Rong Liu* and Bing-Lin Gu \\ Department of Physics, Tsinghua University, Beijing 100084, People's Republic of China \\ Xiao-Wen Zhang \\ Department of Materials Science and Engineering, Tsinghua University, Beijing 100084, People's \\ Republic of China
}

\begin{abstract}
The thermally activated flips of the local spontaneous polarization in relaxors were simulated to investigate the effects of the applied-ac-field amplitude on the dielectric susceptibility. It was observed that the susceptibility increases with increasing the amplitude at low temperatures. At high temperatures, the susceptibility experiences a plateau and then drops. The maximum in the temperature dependence of susceptibility shifts to lower temperatures when the amplitude increases. A similarity was found between the effects of the amplitude and frequency on the susceptibility.

PACS: 77.22.Ch, 77.80.-e, 77.84.-s, 77.84.Lf
\end{abstract}

Typeset using REVTEX 
Relaxor ferroelectrics (relaxors) have been studied for nearly 40 years since $\mathrm{Pb}\left(\mathrm{Mg}_{1 / 3} \mathrm{Nb}_{2 / 3}\right) \mathrm{O}_{3}(\mathrm{PMN})$ was first synthesized by Smolenski et al. $⿴$. The dielectric response of relaxors is characterized by the diffuse phase transition (DPT) and a strong frequency dispersion.2 Various models, such as the compositional heterogeneity model, 1 the superparaelectric model, 目 and the glasslike model, $\frac{3}{3}$ et al., were proposed to rationalize the complicated behaviors of relaxors. It is widely accepted nowadays that the presence of polar microregions in nanoscalet 6 is responsible for the relaxor behaviors.

The effects of the applied ac field on relaxors 14 cause great interest since they provide some clue of the relaxation mechanism. Glazounov et al. observed that the dielectric permittivity of PMN increases with increasing amplitude of the applied ac field A similarity was also found between the effects of the amplitude and frequency on the permittivity. In addition, the ac-drive-enhanced relaxor characteristics and domain breakdown were observed in (PbLa)(ZrTi) (PLZT).11 There are two possible mechanisms, i. e., domain-wall motion model and superparaelectric model, to explicate the nonlinearity of dielectric permitivity of PMN relaxors. Glazounov et al. suggested that it is related to domain-type process rather than thermally activated flips of the local spontaneous polarization (i.e. superparaelectric model). However, they did not consider the interaction of polar microregions when investigating the superparaelectric model, which is just one of the key points related to response of the external field 3 15. 16 . In this study, we conduct a Monte Carlo simulation to investigate the influence of measuring field on the dielectric susceptibility of relaxors.

We investigate the thermally activated flipping process of the local spontaneous polarization in relaxors. Following the work of Gui et al. 15 the polar microregions are regarded as point dipoles. Then relaxors are modeled to be a system consisting of Ising-like dipoles with randomly distributed interactions:国

$$
H=-\sum_{i \neq j} \tilde{J}_{i j} \sigma_{i} \sigma_{j}-E_{\text {ext }} \bar{\mu} \sum_{i} \frac{\left|\mu_{i} \cos \theta_{i}\right|}{\bar{\mu}} \sigma_{i}
$$

where $\sigma_{i}, \sigma_{j}= \pm 1$ are dipole spins. When the projection of the $i$ th dipole moment $\vec{\mu}_{i}$ on the direction of the external field $\vec{E}_{\text {ext }}$ is positive, $\sigma_{i}$ takes value +1 , otherwise, $\sigma_{i}$ takes 
value $-1 . \theta_{i}$ is the angle between $\vec{\mu}_{i}$ and $\vec{E}_{\text {ext }}$, and $\bar{\mu}$ is the maximal magnitude of the dipole moments. $\tilde{J}_{i j}$ is the effective interaction energy between the nearest neighbor dipoles, which has a Gaussian distribution with a width $\Delta J . \tilde{J}_{i j}$ reflects the correlation between polar microregions, which is essential to the glassy behaviors. contains a measuring ac field and a bias dc field. In this paper, only the ac field is involved, i.e.,

$$
E_{\text {ext }}=E_{0} \exp \left(i 2 \pi \frac{t}{t_{L}}\right)
$$

where $t$ is the real time. $E_{0}$ and $t_{L}$ are the amplitude and the period of the ac field, respectively.

The Monte Carlo simulation is performed on a $16 \times 16 \times 16$ simple cubic lattice with periodic boundary conditions. The details of simulation process can be found in Ref. 15. The dielectric susceptibility is defined as

$$
\chi=C\left\langle\frac{\frac{1}{t_{o b s}} \int_{t_{0}}^{t_{0}+t_{o b s}} p(t) \exp \left(i 2 \pi \frac{t}{t_{L}}\right) d t}{E_{\text {ext }}}\right\rangle,
$$

where $C$ is a proportional factor which is chosen to be 1 in this contribution, and $\langle\cdots\rangle$ denotes the configurational averaging. $p(t)$ is the normalized polarization:

$$
p(t)=\frac{1}{N} \sum_{i} \frac{\left|\mu_{i} \cos \theta_{i}\right|}{\bar{\mu}} \sigma_{i} .
$$

During the simulation process, $p(t)$ is recorded and $\chi$ is calculated according to Eq. (3). We choose $t_{0}=200 \mathrm{MCS} /$ dipole to eliminate the influence of the initial state and $t_{o b s}=$ 3000MCS/dipole to be the observation time. The simulation is performed in many runs with different initial conditions so that the configurational averaging can be done. Longer observation time was also adopted in test, but no obvious influence on results was observed.

In order to verify the validity of the method, the dielectric susceptibility under a weak field is firstly calculated. The result is shown in Fig. 1. It can be seen that the susceptibility $\chi$ reaches its maximum at a certain temperature $\left(T_{m}\right)$ and changes gradually around $T_{m}$, which is known as the diffuse phase transition (DPT) in relaxors. A strong frequency dispersion can 
be also observed: $\chi$ decreases with increasing field frequency at low temperatures, and $T_{m}$ moves to higher temperatures. All these characteristics are consistent with the experiments 2 and the previous theoretical results 15 .

Now, let us investigate the effects of the field amplitude on the dielectric susceptibility. The susceptibility curves under different ac-field amplitudes $E_{0}$ are depicted in Fig. 2 when the measuring frequency is kept as $t_{L}=10 \mathrm{MCS} /$ dipole. (We express the frequency by $t_{L}$ here and hereafter.) From Fig. 2 one can list the most essential features of the nonlinear effect: (1) the dielectric susceptibility increases with increasing $E_{0}$ at temperatures $T<T_{m}$ where the frequency dispersion is observed; (2) increasing $E_{0}$ will make the maximum in the temperature dependence of $\chi$ shifts to lower temperatures, which has the similar effect of decreasing frequency (see also Fig. 1). The change of the imaginary part, $\chi^{\prime \prime}$, shows similar features in the simulation. These features agree with the experiments in PMNE月 very well. The concepts of "slow dipole" and "fast dipole" can help to understand the increasing of the susceptibility. Slow dipoles are those dipoles which flip too slow to keep up with the changing of the ac field and give no or little contribution to the dielectric susceptibility. At low temperatures, there are large amounts of slow dipoles.15 When $E_{0}$ increases, the driving force on slow dipoles is enhanced. Slow dipoles are forced to flip faster and they give more contribution to the dielectric susceptibility $\chi$. For fast dipoles, the contribution changes slightly at low drives (see below). As a result, the susceptibility $\chi$ increases with increasing $E_{0}$.

It can be seen in Fig. 2 that the dielectric susceptibility slightly decreases with increasing the external-field amplitude $E_{0}$ at high temperatures. The tendency is weakened at higher frequencies while becomes more evident at lower frequencies. Fig. 3 shows the cases for a lower frequency $t_{L}=50 \mathrm{MCS} /$ dipole. It shows that the dielectric susceptibility decreases at high temperatures and increases at low temperatures when $E_{0}$ increases. These results are similar to the experimental cases in PLZT 1112 to some extent. However, the computed maximum in $\chi(T)$ decreases with increasing $E_{0}$, which is opposite to the experimental observations. 1 It reflects the defect of the model or/and the method we used. 
To get further knowledge of the continuous effects of the ac-field amplitude, we plot in Fig. 4 the curves of $\chi$ as functions of the amplitude $E_{0}$ for different temperatures when the measuring frequency is fixed as $t_{L}=10 \mathrm{MCS} /$ dipole. At low temperatures, the dielectric susceptibility increases first, and then drops with increasing $E_{0}$. This means that the applied field speeds up the flipping of dipoles at small $E_{0}$ values so $\chi$ increases first, while the system is nearly saturated at large $E_{0}$ values which causes the drop of $\chi$. At high temperatures, the dielectric susceptibility experiences a plateau at the beginning and then decreases when the applied field increases. These results are consistent with the experiments in PMN when $E_{0}$ varied in wide range of values.14 In Ref. 7 and Ref. 8, $E_{0}$ is not large enough, so $\chi$ increases at low temperatures and remains steady at high temperatures with increasing $E_{0}$.

Fig. 5 demonstrates the field dependence of $\chi$ at different measuring frequencies and a fixed temperature $T=1.5 \Delta J / k_{B}$. It shows that the maximum of the curve shifts to lower field amplitude when decreasing the measuring frequency. The shapes of curves are similar for different frequencies.

Fig. 6 shows the temperature of the susceptibility maximum $\left(T_{m}\right)$ as a function of the external-ac-field amplitude $E_{0}$. A nonlinear relation can be found between $T_{m}$ and $E_{0}$. It is conflict with the linear law observed in experiments 78 . Perhaps the field used in experiments is not large enough to reveal the high-order effects of the $T_{m} \sim E_{0}$ curve. Further experiments are needed to testify the theoretical predictions.

The Eq. (3) could be generalized to include the Fourier component at different frequencies than that of $E_{\text {ext }}$. Fig. 7 gives the curve of $\chi_{2 \omega} / \chi$, where $\chi_{2 \omega}$ is the second-order component of the susceptibility. It can be seen that $\chi_{2 \omega} / \chi$ is stronger at lower $E_{\text {ext }}$ and $T$.

By means of the results above, we can see that the behaviors of the system described by the model Hamiltonian in Eq. (1) are consistent with many aspects of the experiments when the applied-ac-field amplitude varies. There are two points that should be mentioned here. First, the interactions between polar microregions play an important role in the dielectric response. If the interaction does not exist, the dielectric susceptibility will decrease with increasing field amplitude as what is pointed out by Glazounov et al. 6 8. Secondly, the model 
in Eq. (1) is a rather simplified model. It cannot reflect the effects of external field on the crystal structure completely 11 It describes only the thermally activated flips process of the local polarization. Indeed, there may be more dielectric mechanism in relaxors. For example, It was presented that there may be two kinds of polarization processes in relaxors.17 Very recently, various types of contributions were found to dominate the dielectric response within different ac-drive amplitude ranges.13

In conclusion, the simulation results suggest that the thermally activated flips of the local spontaneous polarization in relaxors plays an important role in producing the relaxation phenomena.

This work was supported by the Chinese National Science Foundation.

* Electronic address: zrliu@phys.tsinghua.edu.cn 


\section{REFERENCES}

${ }^{1}$ G. Smolenski and A. Agranovska, Sov. Phys. Solid State 1, 1429 (1960).

${ }^{2}$ L. E. Cross, Ferroelectrics 76, 241 (1987).

${ }^{3}$ D. Viehland, S. J. Jang, and L. E. Cross, J. Appl. Phys. 68, 2916(1990).

${ }^{4}$ C. Randall, D. J. Barber, R. W. Whatmore, and P. Groves, J. Mater. Sci. 21, 4456 (1987).

${ }^{5}$ X. H. Dai, Z. Xu, and D. Viehland, Philos. Mag. B 70, 33 (1994).

${ }^{6}$ F. Fang and X. W. Zhang, J. Mater. Sci. 3, 1495 (1996).

${ }^{7}$ A. E. Glazounov, A. K. Tagantsev, and A. J. Bell, Phys. Rev. B 53, 11281 (1996).

${ }^{8}$ A. E. Glazounov, A. K. Tagantsev, and A. J. Bell, Ferroelectrics 184, 217 (1996)

${ }^{9}$ A. K. Tagantsev, A. E. Glazounov, Phys. Rev. B 57, 18 (1998)

${ }^{10}$ A. E. Glazounov, A. K. Tagantsev, J. Phys.: Condens. Matter. 10, 8863 (1998)

${ }^{11}$ Q. Tan and D. Viehland, J. Am. Ceram. Soc. 79, 2747 (1996).

${ }^{12}$ Q. Tan and D. Viehland, Phys. Rev. B 53, 14103 (1996)

${ }^{13}$ E. V. Colla, E. L. Furman, S. M. Gupta, N. K. Yushin, and D. Viehland, J. Appl. Phys. 85, $1693(1999)$

14 T. Tsurumi, K. Soejima, T. Kamiya, and M. Daimon, Jpn. J. Appl. Phys. 33, 1959 (1994)

${ }^{15}$ H. Gui, B. L. Gu, and X. W. Zhang, Phys. Rev. B 52, 3135 (1995).

${ }^{16}$ D. Viehland, J. F. Li, S. J. Jang, L. E. Cross, and M. Wutting, Phys. Rev. B 46, 8013 (1992).

${ }^{17}$ Z. -Y. Cheng, R. S. Ratiyar, X. Yao, and A. S. Bhalla, Phys. Rev. B 57, 8166 (1998). 


\section{FIGURES}

FIG. 1. Weak-field susceptibility as a function of temperature (in units of $\Delta J / k_{B}$ ). The field amplitude is fixed as $E_{0}=0.1 \Delta J / \bar{\mu}$. The curves 1-4 correspond to the field frequency $t_{L}=100,50,20,10 \mathrm{MCS} /$ dipole, respectively.

FIG. 2. Dielectric susceptibility at various field amplitudes, $E_{0}(1-0.5,2-1.0,3-1.5,4-2.0 \Delta J / \bar{\mu})$. The field frequency is kept as $t_{L}=10 \mathrm{MCS} /$ dipole. Inserted graphics is the imaginary part of susceptibility.

FIG. 3. Dielectric susceptibility at various field amplitudes, $E_{0}(1-0.5,2-1.0,3-1.5,4-2.0 \Delta J / \bar{\mu})$. The field frequency is kept as $t_{L}=50 \mathrm{MCS} /$ dipole.

FIG. 4. Field amplitude (in units of $\Delta J / \bar{\mu}$ ) dependence of dielectric susceptibility at a fixed field frequency $t_{L}=10 \mathrm{MCS} /$ dipole. Curves $1-5$ correspond to temperatures $\mathrm{T}=0.5,1.5,2.5,3.5$ and $4.5 \Delta J / k_{B}$, respectively.

FIG. 5. Field amplitude dependence of susceptibility at various frequencies and a fixed temperature $T=1.5 \Delta J / k_{B}$. The amplitude and the frequency are measured in units of $\Delta J / \bar{\mu}$ and MCS/dipole, respectively.

FIG. 6. Temperature $T_{m}$, corresponding to the position of the maximum in $\chi(T)$, as a function of the field amplitude (in units of $\Delta J / \bar{\mu}$ ). The temperature is measured in units of $\Delta J / k_{B}$.

FIG. 7. Temperature dependence of $\chi_{2 \omega} / \chi$ at various field amplitudes, $E_{0}(1-0.5,2-1.5 \Delta J / \bar{\mu})$. The field frequency is kept as $t_{L}=10 \mathrm{MCS} /$ dipole. 


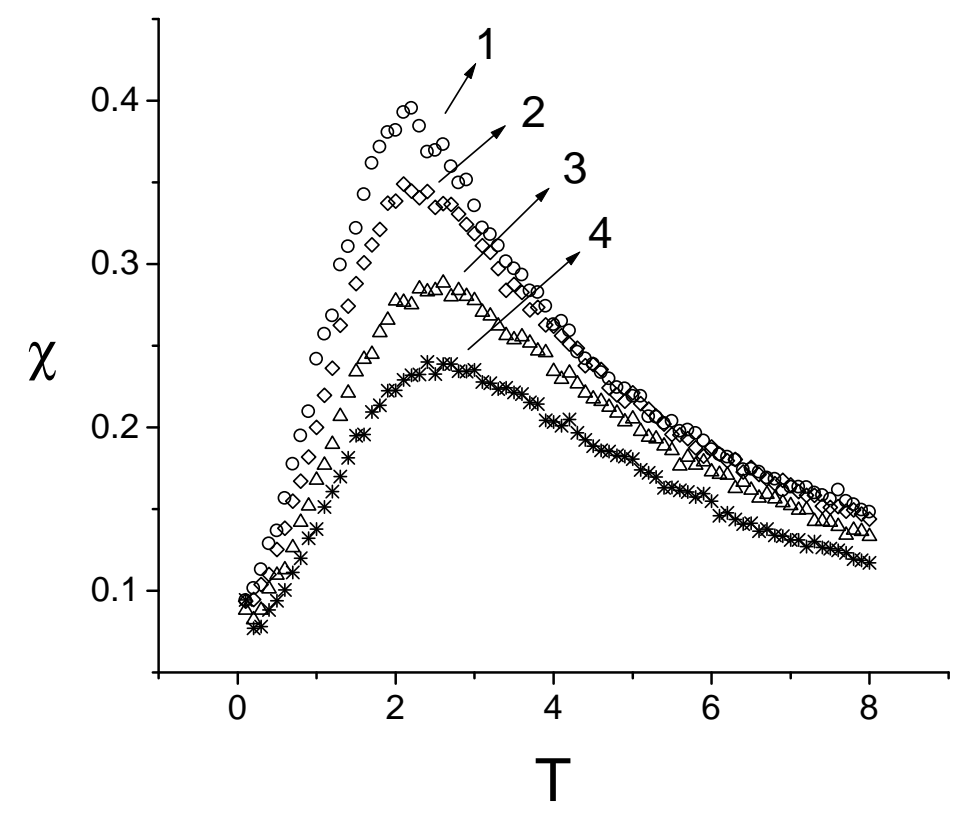




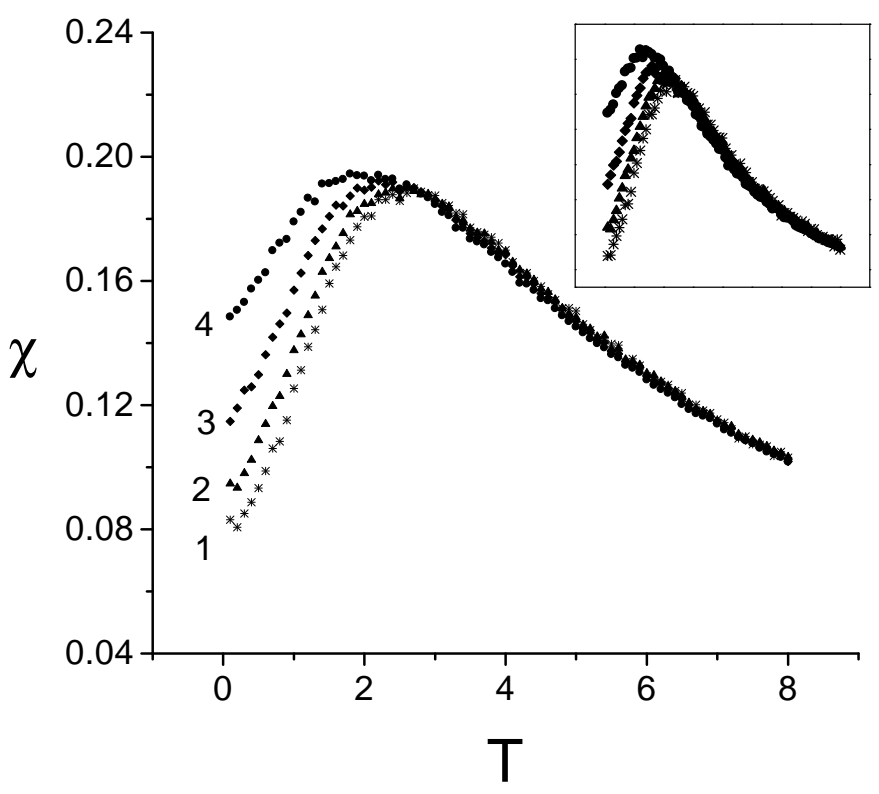




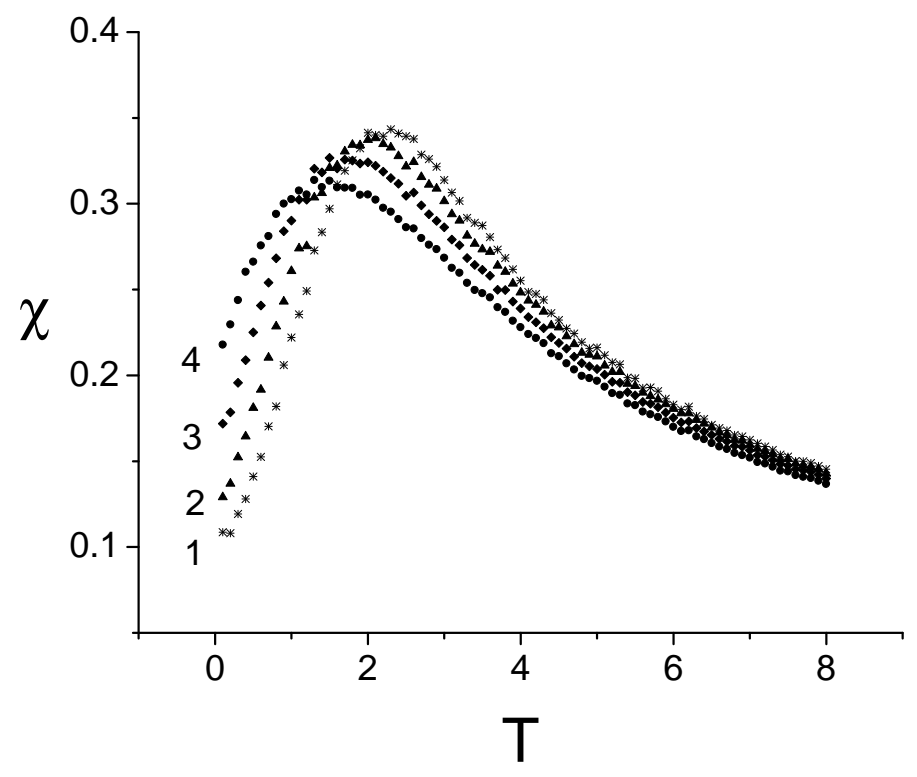




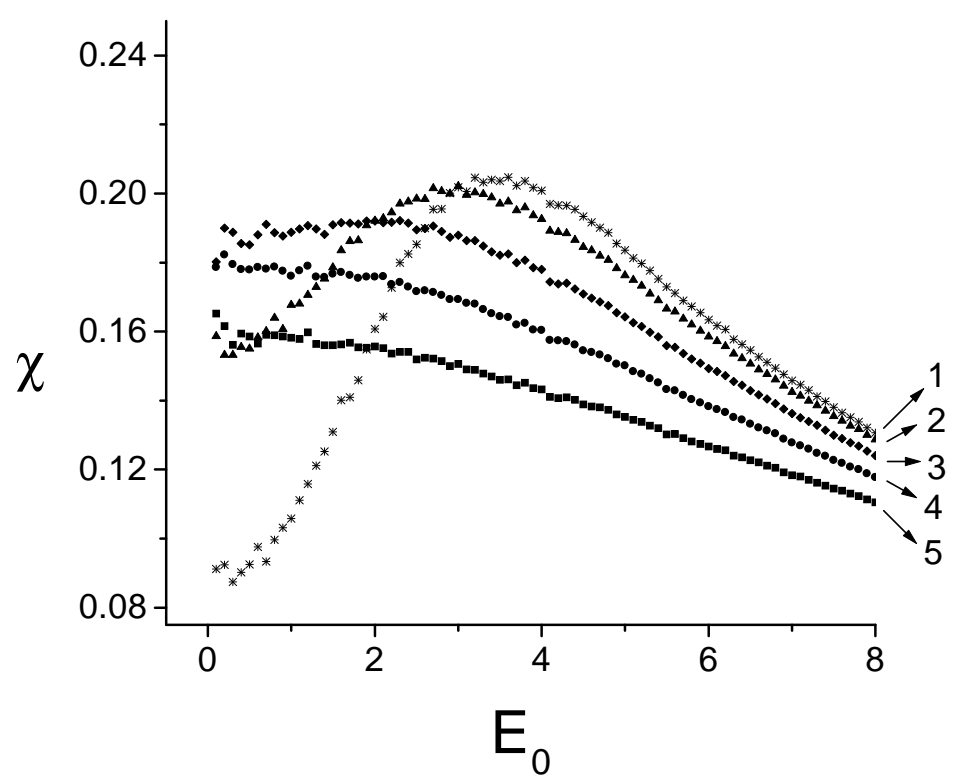




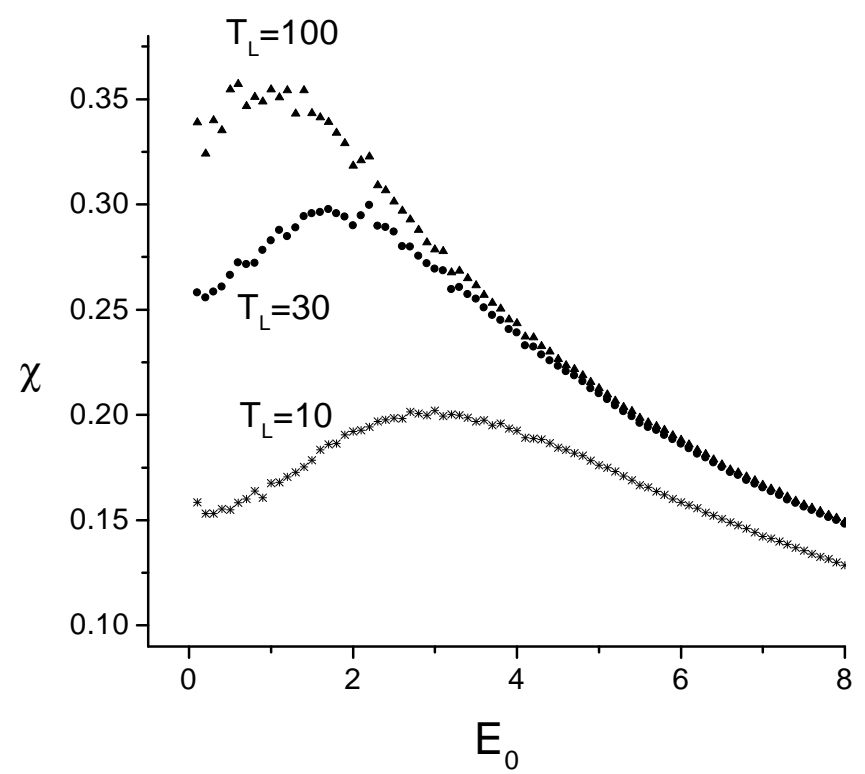




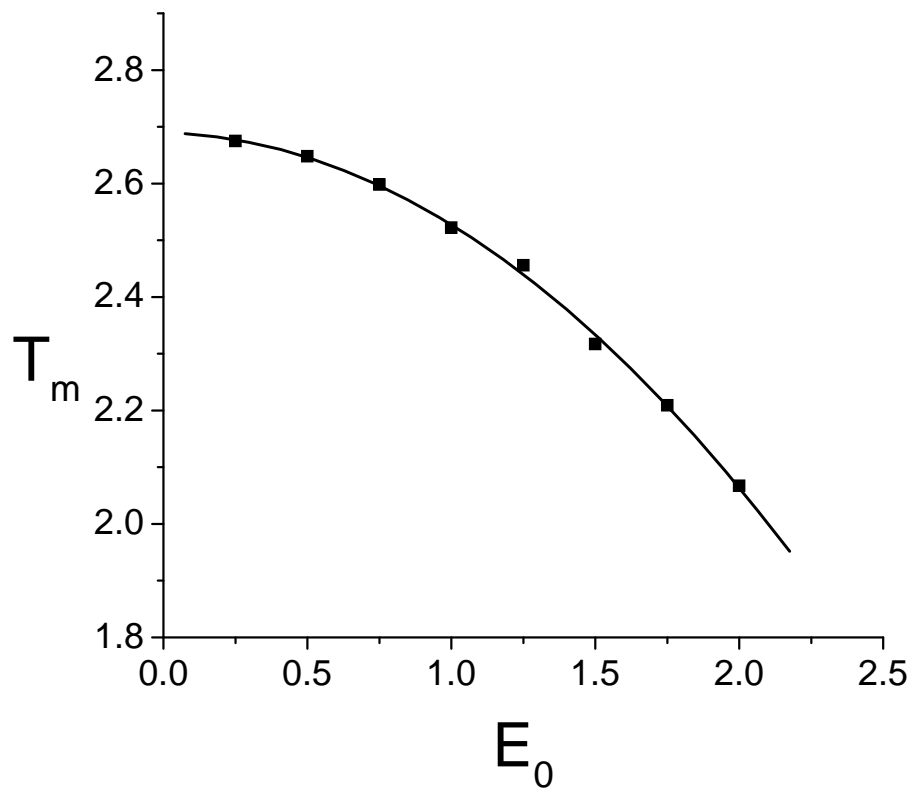




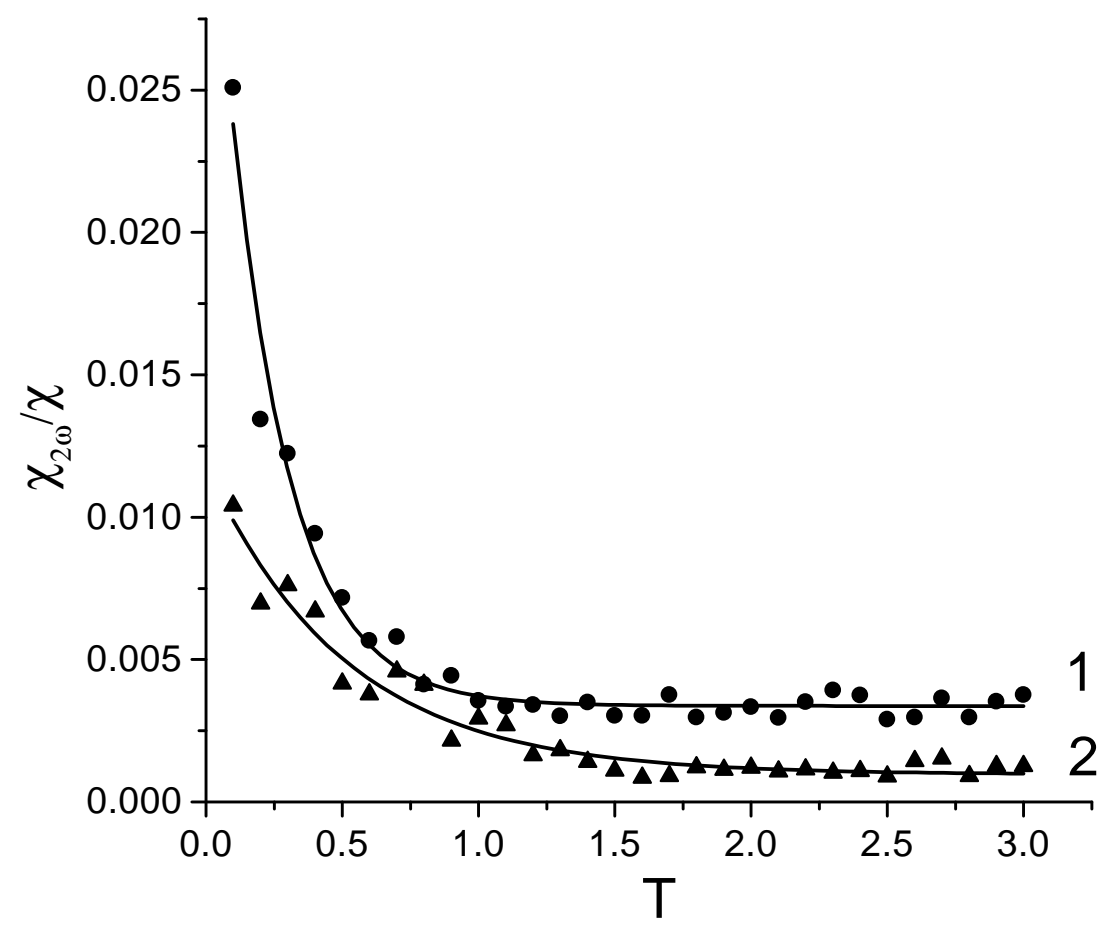

